\title{
Redistributing working schedules using the infective principle in the response to COVID-19
}

\author{
Marko Ćurković MD, $\mathrm{PhD}^{1,2}$, Andro Košec MD, $\mathrm{PhD}^{2,3}$ (1) and Petrana Brečić MD, $\mathrm{PhD}^{1,2}$ \\ ${ }^{1}$ University Psychiatric Hospital Vrapče, Zagreb, Croatia, ${ }^{2}$ School of Medicine, University of Zagreb, Zagreb, Croatia and ${ }^{3}$ Department of Otorhinolaryngology and \\ Head and Neck Surgery, University Hospital Center Sestre milosrdnice, Zagreb, Croatia
}

To the Editor-Many healthcare professionals are currently witnessing, or being actively involved in dealing with, the COVID-19 global pandemic and its tragic pathways and outcomes. ${ }^{1,2}$ The battle against this pandemic is putting enormous pressure on healthcare systems worldwide, primarily due to the infectiousness of the infective agent and the specific health consequences it causes. ${ }^{2,3}$ Most public health measures are aimed at delaying the peak pandemic effects and preventing the overflow and subsequent collapse of healthcare systems. ${ }^{2,3}$ Although this pandemic has been widely reported on and discussed for several months, healthcare systems seems to be ill prepared, putting healthcare professionals at the center of complex personal, professional, and societal demands. ${ }^{1,3-5}$ Under extreme pressure, many healthcare professionals may express various psychological problems, such as exhaustion, fatigue, burnout, anxiety, and depression, all of which can undermine their ability to care for their health and safety, as well as that of their colleagues, their close ones, and their patients. ${ }^{4,5}$ Although this problem is especially prevalent among "frontline" healthcare professionals, other professionals may be affected as the pandemic spreads and affects competing interests pertaining to non-pandemic-related health issues. ${ }^{4,5}$ Many proposals have been put forth regarding how to prevent and mitigate these possible adverse effects, such as the introduction of different procedures and guidelines, the availability of instrumental and psychological support, and more appropriate staffing and shift scheduling. ${ }^{5-8}$

As the consequences of advanced pandemics become more evident, even with certain restrictive public health measures in place, the continuity of care for other health issues must be preserved because disrupted availability and access to care in pandemic settings may drive non-pandemic-related mortality. ${ }^{2,6,9}$ Therefore, it is vitally important to control the introduction and spread of SARS-CoV-2 in all healthcare units and institutions that provide care for noninfected patients, so they remain COVID-19 free. ${ }^{6,8,9}$ The emergence of infection in these settings may be particularly difficult to deal with because it may affect vulnerable populations as it rapidly continues to spread. ${ }^{2,6-9}$ Furthermore, this pandemic may further disrupt the availability of care for nonpandemic conditions. ${ }^{3,6}$ This factor is of special relevance in units and institutions dealing with health conditions considered significant risk factors for severe COVID-19 disease.

In these units, among other widely used measures (eg, vigorous screening for possible COVID-19 infection, stringent testing, and triage of suspected cases as well as developing and adopting different

Author for correspondence: Marko Ćurković, E-mail: markocurak@gmail.com Cite this article: Ćurković M, Košec A, and Brečić P. (2020). Redistributing working schedules using the infective principle in the response to COVID-19. Infection Control \& Hospital Epidemiology, 41: 1123-1124, https://doi.org/10.1017/ice.2020.155 care, safety, allocation, and communication strategies), it is of immense importance in controlling possible transmission of infection among and by healthcare professionals. Healthcare professionals may remain unaware of their COVID-19 status due to the mild or even asymptomatic course in healthy individuals; thus, they may unknowingly become supervectors. ${ }^{6-8,10}$ Healthcare settings are usually inadequately prepared for infection prevention and control, especially in the context of limited resources (eg, personal protective equipment), and professionals may become infected through their contact with even seemingly noninfected patients. In addition to previously reported measures, alternative distribution of working schedules may contribute to minimizing the likelihood of virus transmission.

In centers where infected patients are treated, healthcare staff are usually organized in 2-week shifts and then spend the next 2 weeks in self-isolation, preferably being (re)tested before starting a new shift. This approach to staff scheduling seems logical because it follows the COVID-19 incubation period.

Work schedules in "COVID-free" institutions should be organized such that after 1 shift (preferably a 12-hour or, exceptionally, a 24-hour shift), healthcare staff remain in self-isolation for 48 hours. Such a redistribution of shifts makes it possible to resolve the nature of possible infective exposure because the COVID-19 infection window seems to be 48 hours (ie, patients become contagious 48 hours before the onset of symptoms). If a healthcare professional comes into close contact with a patient who later develops symptoms, it is prudent to automatically prolong the self-isolation period until the COVID-19 status of that patient is resolved, allowing enough time to contain possible events of disease transmission. Such a redistribution of working hours, together with rigorous tracking of any relevant close contacts (which could be assisted by novel technologies) limits possible introduction and spread of infection within the institution.

This approach may have special importance in the pandemic timeline. When measures of self-isolation and/or quarantine are in place and local disease transmission has been demonstrated, clinical spread of infection will become the most important. Furthermore, since healthcare professionals among the few persons allowed free movement under "stay at home" orders, this scheduling strategy may have important repercussions for the entire community because it can limit the potential pandemic vector effect, which not yet well understood. $2,6,10$

This scheduling approach may be feasible in institutions with sufficient staff to maintain a work schedule of continuous rotation, which may be difficult, but it may prove useful in the long run. Additionally, through alternative methods of care delivery, like those provided through novel digital technologies, staff that are not physically present may remain fully involved in providing 
care for those who need it most. Finally, this scheduling approach may preserve the bulk of physically and mentally healthy staff sorely needed to combat later effects of pandemic, which will have dire consequences for healthcare systems that have not made every effort to prevent intrainstitutional transmission.

\section{Acknowledgments. None.}

Financial support. No financial support was provided relevant to this article.

Conflicts of interest. M.Ć. and P.B. have received lecture honoraria from Lundbeck, Sandoz, Janssen, Pliva (Teva), and Alkaloid. A.K. has no conflicts of interest to declare.

\section{References}

1. Wu Z, McGoogan JM. Characteristics of and important lessons from the coronavirus disease 2019 (COVID-19) outbreak in China. JAMA 2020 [Epub ahead of print]. doi: 10.1001/jama.2020.2648.

2. Hick JL, Biddinger PD. Novel coronavirus and old lessons-preparing the health system for the pandemic. N Engl J Med 2020 [Epub ahead of print]. doi: 10.1056/NEJMp2005118.

3. Emanuel EJ, Persad G, Upshur R, et al. Fair allocation of scarce medical resources in the time of COVID-19. N Engl J Med 2020 [Epub ahead of print]. doi: 10.1056/NEJMsb2005114.
4. Lima CKT, Carvalho PMM, Lima IAAS, et al. The emotional impact of coronavirus 2019-nCoV (new Coronavirus disease). Psychiatry Res 2020;287:112915 [Epub ahead of print]. doi: 10.1016/j.psychres.2020. 112915.

5. Greenberg N, Docherty M, Gnanapragasam S, Wessely S. Managing mental health challenges faced by healthcare workers during covid-19 pandemic. BMJ 2020 Mar 26;368:m1211. doi: 10.1136/bmj.m1211.

6. Chopra V, Toner E, Waldhorn R, Washer L. How should US hospitals prepare for coronavirus disease 2019 (COVID-19)? Ann Intern Med 2020 Mar 11 [Epub ahead of print]. doi: 10.7326/M20-0907.

7. Xiang YT, Zhao YJ, Liu ZH, et al. The COVID-19 outbreak and psychiatric hospitals in China: managing challenges through mental health service reform. Int J Biol Sci 2020 Mar 15;16:1741-1744.

8. Chang, Xu H, Rebaza A, Sharma L, Dela Cruz CS. Protecting health-care workers from subclinical coronavirus infection. Lancet Respir Med 2020; 8(3):e13. doi: 10.1016/S2213-2600(20)30066-7.

9. Bearman G, Pryor R, Albert H, et al. Novel coronavirus and hospital infection prevention: preparing for the impromptu speech. Infect Control Hosp Epidemiol 2020;3:1-2 [Epub ahead of print]. doi: 10.1017/ ice.2020.55.

10. Gaur S, Dumyati G, Nace DA, Jump RLP. Unprecedented solutions for extraordinary times: helping long-term care settings deal with the COVID-19 pandemic. Infect Control Hosp Epidemiol 2020;30:1-8 [Epub ahead of print]. doi: 10.1017/ice.2020.98.

\title{
Bacterial and fungal infections in COVID-19 patients: A matter of concern
}

\author{
Pengcheng Zhou MD ${ }^{1,2}$, Zhenguo Liu MD², Yuhua Chen MSN ${ }^{1}$, Yinzong Xiao MD ${ }^{1,3}$, Xun Huang MD ${ }^{1}$ and Xue-Gong Fan MD \\ ${ }^{1}$ Xiangya Hospital, Central South University, Changsha, China, ${ }^{2}$ The Third Xiangya Hospital, Central South University, Changsha, China and ${ }^{3}$ Burnet Institute, \\ St Vincent's Hospital Melbourne, and University of Melbourne, Melbourne, Australia
}

To the Editor-Coronavirus disease 2019 (COVID-19) has become a pandemic. As of April 2, 2020, a total of 896,450 laboratoryconfirmed cases have been reported. The death toll from COVID-19 has soared quickly: 45,526 deaths have been reported globally, including 24,692 deaths in only a week (March 26, 2020, through April 2, 2020). ${ }^{1}$ Age, sequential organ failure assessment (SOFA) score, and D-dimer are the main prognostic factors of COVID-19 patients. ${ }^{2}$ The presence of bacterial and/or fungal secondary infection or coinfection is likely another important factor affecting mortality, and it has received inadequate attention.

Bacterial and fungal infections are common complications of viral pneumonia, especially in critically ill patients. They lead to increased need for intensive care and increased mortality. In influenza patients, bacterial coinfection occurs in $\sim 0.5 \%$ of healthy young individuals and at least $2.5 \%$ of older individuals. ${ }^{3} \mathrm{~A}$ systematic review revealed that $\sim 1$ in 4 H1N1 patients during the 2009 pandemic had a bacterial or fungi infection infection. ${ }^{4}$ Data regarding the bacterial or fungi infection in viral pneumonia led by

Author for correspondence: Xue-Gong Fan MD Or Xun Huang MD, E-mail: xgfan@ hotmail.com or huangxun224@126.com.

Cite this article: Zhou P, et al. (2020). Bacterial and fungal infections in COVID-19 patients: A matter of concern. Infection Control \& Hospital Epidemiology, 41: 1124-1125, https://doi.org/10.1017/ice.2020.156 coronavirus are limited. According to the cohort study report by Zhong Nanshan et al, ${ }^{5} 20$ of 90 severe acute respiratory syndrome (SARS) patients had secondary lower respiratory tract infections in 2003 , which accounted for $70.6 \%$ of those critical SARS patients who underwent an invasive operation. The pathogens causing secondary infections in SARS patients were diverse: negative bacilli were the most common but Candida was also common. ${ }^{5}$ Invasive pulmonary aspergillosis was another common complication secondary to influenza. ${ }^{6}$

Bacterial and fungal infections in COVID-19 patients have been inadequately investigated and reported thus far. Among the hundreds of articles published with clinical data, only a few have reported secondary infection, mostly without detailed pathogens (Table 1). Even in studies for which secondary infection data are available, the antibiotics use rate $(94 \%-100 \%)$ was much higher than the reported incidence of secondary infection $(10 \%-15 \%)^{2,7,8}$ In addition, the complication of bacterial or fungal infection was not included in the prognosis analysis in most published papers. Moreover, most of the current infection control protocols aim to prevent the transmission and cross infection by SARS-CoV-2, missing the prevention of bacterial or fungal secondary infection. In fact, secondary infection was found in $50 \%$ of nonsurviving COVID-19 patients. $^{2}$

Thus far, many diagnostic and prevention approaches to targeting complications in COVID-19 patients have been outlined in

(c) 2020 by The Society for Healthcare Epidemiology of America. All rights reserved. This is an Open Access article, distributed under the terms of the Creative Commons Attribution licence (http://creativecommons.org/licenses/by/4.0/), which permits unrestricted re-use, distribution, and reproduction in any medium, provided the original work is properly cited. 